\title{
Compared sensitivities of VLT, JWST and ELT for direct exoplanet detection in nearby stellar moving groups
}

\author{
Charles Hanot $^{a}$, Olivier Absil ${ }^{a}$, Jean Surdej ${ }^{a}$, Anthony Boccaletti ${ }^{b}$ and Christophe Vérinaud $^{c}$ \\ ${ }^{a}$ Institut d'Astrophysique et de Géophysique, University of Liège, 17 allée du 6 Août, B-4000, \\ Sart Tilman, Belgium; \\ ${ }^{b}$ LESIA, Observatoire de Meudon, 5 pl. J. Janssen, 92195, Meudon, France; \\ ${ }^{c}$ LAOG, 414 rue de la Piscine, Domaine Universitaire, 38041, Grenoble, France
}

\begin{abstract}
In the context of exoplanet detection, a large majority of the 400 detected exoplanets have been found by indirect methods. Today, progress in the field of high contrast and angular resolution imaging has allowed direct images of several exoplanetary systems to be taken (cf. HR 8799, Fomalhaut and $\beta$ Pic). ${ }^{1-4}$ In the near future, several new instruments are going to dramatically improve our sensitivity to exoplanet detection. Among these, SPHERE (Spectro Polarimetric High contrast Exoplanet REsearch) at the VLT, MIRI (Mid Infra-Red Instrument) onboard JWST and EPICS at the ELT will be equipped with coronagraphs to reveal faint objects in the vicinity of nearby stars. We made use of the Lyon group (COND) evolutionary models of young (sub-)stellar objects and exoplanets to compare the sensitivities of these different instruments using their estimated coronagraphic profiles. From this comparison, we present a catalogue of targets which are particularly well suited for the different instruments.
\end{abstract}

Keywords: Coronagraphy, Exo-planets, Moving-groups

\section{INTRODUCTION}

Extrasolar planets are being nowadays routinely detected by radial velocity and transit surveys around G-K dwarfs, ${ }^{5}$ while first significant results on early and late type dwarfs are starting to emerge. ${ }^{5,6}$ The statistical study of the distribution of planets has considerably changed our view of extrasolar planetary systems. Extending the planet search towards sub-Jupiter mass planets at long periods in a statistically meaningful way would provide additional constraints on planet forming scenarios (e.g., core accretion vs. disk instability), including the role of planetary migration. Direct imaging is a very appropriate technique to extend this statistical study for planets with long periods and for all stellar spectral types.

Young main sequence stars hold a key place in the study of planetary systems with direct imaging. With ages typically between 10 and $100 \mathrm{Myr}$, the optically thick protoplanetary discs originally surrounding these stars have been cleared out by various physical processes and only optically thin disks are still present. Therefore, it provides a direct view of young planetary systems. At such ages, giant planets have just formed and have not yet had the time to cool down to their final equilibrium temperature. They are thus significantly brighter than their old counterparts. ${ }^{7,8}$ Theoretical models for the early cooling and contraction of giant planets are however poorly constrained, and high uncertainties are affecting the first tens of Myr in their cooling history. In particular, the sensitivity to initial conditions is strongly affecting the cooling models during the first 10-100 Myr for planets with masses larger than that of Jupiter (see Figure 1). The direct detection and characterisation of extrasolar planets with well-defined ages below $100 \mathrm{Myr}$ therefore represent a much-needed observational constraint for these models.

During the past decade, a series of young stellar kinematic groups have been identified in the solar neighbourhood $(<100 \mathrm{pc})$, with ages ranging from about 8 to $200 \mathrm{Myr} .{ }^{10}$ Searching for planets around such stars has become a very active topic in extrasolar planet studies during the past decade. Dedicated observing campaigns have been undertaken in all major observatories (especially with 10-m class telescopes), and the forthcoming advent of 2nd generation planet imaging instruments equipped with extreme adaptive optics (such as VLTI/SPHERE,

Further author information: (Send correspondence to Charles Hanot) Charles Hanot: E-mail: hanot@astro.ulg.ac.be, Telephone: +32 (0)4 3669768 

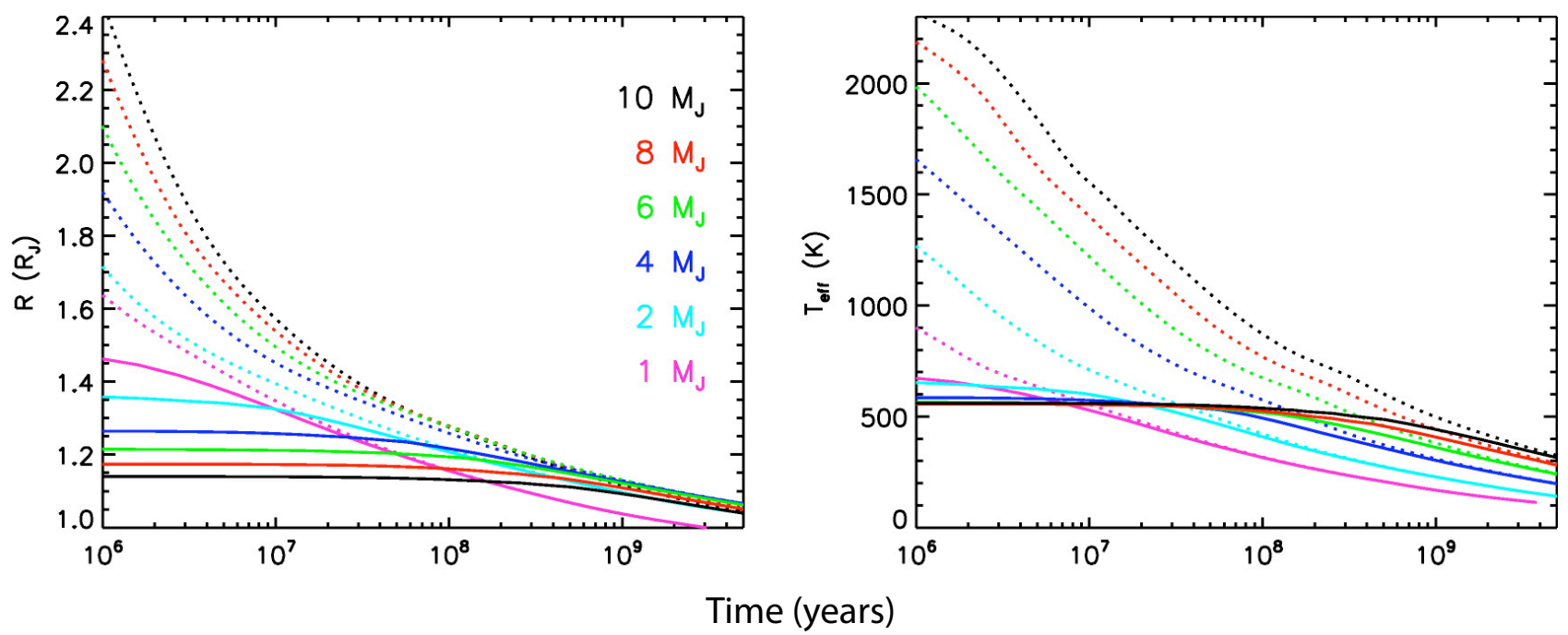

Figure 1. Planetary thermal evolution models from Fortney et al. 2008 (solid lines, core-accretion model) and from Baraffe et al. 2003 (dotted line, hot start model), showing planetary radius and temperature as a function of time (in yr). From Fortney et al. 2008. ${ }^{9}$

Gemini/GPI and Subaru/HiCIAO) will further improve the sensitivity of ground-based telescopes to giant planets in nearby young associations, especially for semi-major axes in the 5-100 AU range (where most giant planets are supposed to be found). However, such instruments will achieve their ultimate performance only on relatively bright stars $(\mathrm{V} \leq 10)$. For fainter stars, the $\mathrm{AO}$ systems will generally fail to provide high-quality wave front correction due to the limited sensitivity of wave front sensors. Consequently, ground- and space-based imaging instruments will be very complimentary as they will have optimal performances for different type of stars. MIRI/JWST will provide a tremendous sensitivities to giant planets around M dwarfs in nearby associations, which have $\mathrm{V}>10$ in almost all cases while ground-based facilities will give the full potential of their high angular resolutions around young and bright stars.

Current RV and transit surveys are detecting planets with orbital distances below $5 \mathrm{AU}$, and larger separations (5-100 AU) are still to be explored. Improving our understanding of planetary formation at such distances will also bring much-needed constraints on the formation of terrestrial planets in the habitable zone of such stars, which are expected to be prime targets for the search of bio-signatures. In this context, the new high contrast imagers have a decisive role to play, because they provide both the sensitivity and the angular resolution to unveil a whole new population of low-mass planets in the 0.5-100 AU region around nearby young stars.

\section{INSTRUMENTAL OVERVIEW}

The direct imaging of exoplanetary systems is a difficult task to accomplish because of both the small angular separation between the planet and its star and the high contrast ratio between them. To do so, it is therefore mandatory to use large telescopes working at their diffraction limit and to dim the strong on-axis starlight to reveal the presence of a planet. Actual telescopes are limited to the imagery of giant planets at relatively large orbital distances. Indeed, on one side, space-based telescopes are limited by the size of their apertures and therefore by their resolution. On the other side, ground based telescopes dispose of larger apertures but are limited by the atmospheric turbulence that blurs the images and decreases the sensitivity of high contrast imaging instruments. Before the end of the decade, a new generation of ground and space based facilities will come online and dramatically increase the sensitivity of exoplanetary imaging and spectroscopy. Among the ground based imagers let us cite SPHERE on the VLT, ${ }^{11}$ GPI on Gemini South, ${ }^{12}$ HiCiAO at Subaru, Project 1640 at Palomar observatory ${ }^{13}$ but also the future extremely large telescopes such as the Thirty Meter Telescope (TMT), ${ }^{14}$ the Giant Magellan Telescope (GMT) ${ }^{15}$ and the European Extremely Large Telescope (E-ELT). ${ }^{16}$ The spaceborne planetary imagers will be led by the James Webb Space Telescope equipped with the Mid InfraRed Instrument 


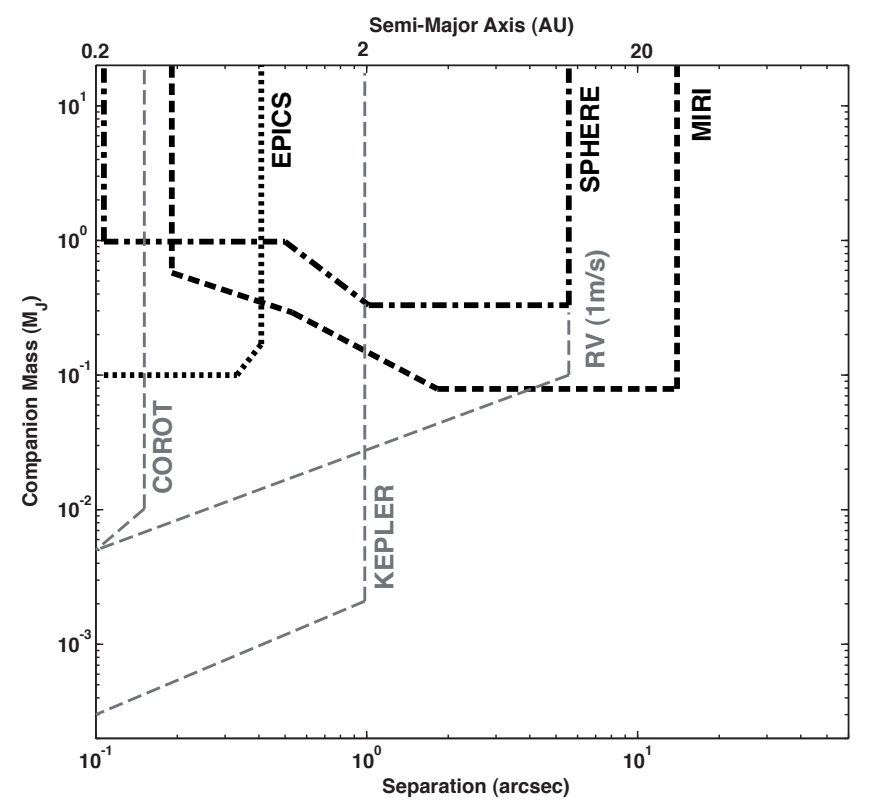

Figure 2. This graph compare the sensitivity of different instruments for exoplanet detection as a function of the separation between the star and its planet. For MIRI, SPHERE and EPICS, the results correspond to the observation of young closeby stars $(10 \mathrm{Myr})$. Indeed it is for these particular stars that those instruments are the most sensitive. The integration time has been set to $1 \mathrm{~h}$. For the three other curves (COROT, KEPLER and Radial Velocities), the sensitivities correspond to observations of exoplanets around main sequence stars.

$(\mathrm{MIRI}),{ }^{17}$ the Near InfraRed Camera (NIRCam) ${ }^{18}$ and the Tunable Filter Imager (TFI). ${ }^{19}$ In this paper, we particularly focus on the comparison of three of these instruments that can be considered as representative of the three categories: SPHERE/VLT and EPICS/E-ELT for the ground-based instruments and MIRI onboard JWST.

\subsection{Description of the different instruments}

In order to describe the main technical particularities of the three instruments that we envision to compare, let us split these instruments into two natural categories: the ground and the space-based telescopes.

\subsubsection{Ground based instruments}

Ground based telescopes have to face a major problem in order to perform high contrast imaging: the Earth atmosphere. The atmosphere indeed strongly limits the possibilities for exoplanet detection for two reasons. First, the introduced turbulence prevents the telescope from producing sharp, diffraction limited images and limits the achievable contrast as the PSF residuals of the central star are spread over a large area in the field of view. Second, ground-based observatories are limited to wavelengths smaller than typically $\sim 4 \mu \mathrm{m}$. For longer wavelengths, even though some ground based imagers exist, the thermal background strongly limits their sensitivities. On earth, we are therefore limited to wavelength ranges across which the exoplanet/star contrast is not very favorable. Indeed, for an Earth-like exoplanet around a solar type star, the contrast is $10^{-10}$ in the visible and near-IR while it is "only" $10^{-7}$ in the thermal infrared.

To correct for wavefront errors introduced by the atmospheric turbulence, many developments have been done in the field of Adaptive Optics (AO). With a high density of actuators (1600 actuators for the 8m telescope of SPHERE and 44521 actuators for the $42 \mathrm{~m}$ telescope of EPICS), both SPHERE and EPICS will be able to use the full potential of their large apertures as they will reach the so called Extreme AO (XAO) regime with Strehl ratios $\geq 90 \%$. With such a sharp image on large telescopes, these two imagers will be able to reach very high contrasts down to $\sim 10^{-6}$ for SPHERE in both $\mathrm{H}$ and $\mathrm{K}$ bands and even $10^{-9}$ for EPICS $[0.95,1.65] \mu \mathrm{m}$. To reach 
such contrasts, they will make use of high contrast imaging techniques to strongly dim the starlight. SPHERE will be equipped with Four Quadrant Phase Mask Coronagraphs and Apodized Lyot Coronagraphs (APLC) ${ }^{20-23}$ while the current baseline of EPICS design considers the use of a simple apodizer to mitigate the chromaticity effects. ${ }^{24,25}$ These systems are combined to differential imaging techniques (spectral, polarimetric and angular) to reach the desired level of contrast. Their discovery space, defined by the Inner Working Angle (IWA) and by the outer Field of View (FOV) of the instruments are respectively [0.1", 5.5"] (limited by the detector FOV) for SPHERE and [0.02", 0.4"] for EPICS. Figure 2 illustrates the sensitivity of the two instruments inside their respective FOV. The price to pay to get such an image quality despite the atmospheric turbulence is a relative lack of sensitivity. Indeed, in order to leave very small wavefront error residuals, AO systems must be fed by relatively bright stars with visual magnitudes $V \leq 10$.

\subsubsection{Space-based instruments}

Space-based telescopes have completely different constraints. The main limitation comes from the size of the telescope that can fit inside a launcher. JWST will be the largest scientific telescope ever launched with a 6.5 $\mathrm{m}$ segmented mirror. The MIRI instrument proposes 4 different wavebands for its coronagraphic mode that are respectively centered on $10.65,11.4,15.5$ and $23 \mu \mathrm{m}$. While the latter channel will be equipped with a standard Lyot coronagraph, the three other ones will use FQPM coronagraphs, which compensate for the lack of angular resolution compared to ground-based facilities. ${ }^{26,27}$ Indeed, such coronagraphs have a much smaller IWA than standard Lyot coronagraphs. For the three "short" wavelength channels, the discovery space will be comprised between $\sim 0.2$ " and $\sim 13$ " (see Fig. 2). The strong point of MIRI is of course its sensitivity to low-mass companions as it operates at thermal infrared wavelengths with low background levels, where warm planets are bright.

\section{SENSITIVITY ESTIMATION}

\subsection{Simulations}

Our simulations are based on a three step process:

- We choose young stars with known ages among the closest young moving groups.

- Using the coronagraphic profiles of the different instruments as a function of the angular separation of the companion and of the brightness of the source, we derive the contrast that can be achieved on each selected target.

- Knowing the age and brightness of the sources as well as the achievable contrasts, we derive the corresponding masses of the companions using the COND evolutionary models.

The goal of this study is to compare the three instrumental performances under simple conditions and assumptions. These assumptions must be chosen to obtain a fair comparison between the three instruments, even though they do not use the same technologies. As for the integration time we adopt a generic value of $1 \mathrm{~h}$ even though in the reality this can be modulated depending on the instrument and the star magnitude. Longer integrations will be needed for fainter stars and/or to reach larger contrasts as long as the systematics remain

low. However, for surveys on such instruments under high scientific pressure, it is probably not realistic to consider $10 \mathrm{~h}$ of observation for dozens of stars. As a comparison, only the relative sensitivities do matter. The other assumptions are the following:

- For MIRI, we use for the coronagraphic profile, contrasts corresponding to the use of the FQPM at 11.4 $\mu \mathrm{m}$ and a simple reference subtraction. The reason for choosing this wavelength is because there is less chance to see the planetary signal being dimmed by an absorption line (cf. Ammonia at $10.65 \mu \mathrm{m}$ ) and the angular resolution is significantly better than at $15.5 \mu \mathrm{m}$.

- On SPHERE, we use two different hypotheses: (i) the use of a FQPM in H band (1.68 $\mu \mathrm{m})$ and a simple reference subtraction (ii) on top of the reference subtraction we use Spectral Differential Imaging ${ }^{28}$ between $^{2}$ the $\mathrm{H} 2(1.593 \mu \mathrm{m})$ and $\mathrm{H} 3(1.667 \mu \mathrm{m})$ spectral bands. ${ }^{29}$ 
- EPICS has unfortunately relatively different hypotheses from the two other instruments. It is due to the way the instrument is conceived and simulated. Indeed, by default, EPICS uses more advanced subtraction methods such as SDI, Angular Differential Imaging (ADI) and differential polarimetry. The instrument uses wavelengths between 0.95 and $1.65 \mu \mathrm{m}$.

Finally, the instrumental simulations are not the only component of our study. Indeed, in order to calculate the masses corresponding to a given magnitude at a given age, we used planetary evolutionary models. Two categories of models are currently available (i) the so called "hot start" cooling models ${ }^{7,30}$ and (ii) the "core accretion" evolutionary models. ${ }^{9}$ These two types of models show discrepancies up to a few magnitudes during the first 10-100 Myr after their formation (see Figure 1). In our simulations, we choose the "hot start" cooling models ${ }^{7,30}$ as these are available for planet masses $<1 \mathrm{M}_{\mathrm{Jup}}$. In order to account for a realistic planetary composition, we use the $\mathrm{Z}=0.1$ models (where $\mathrm{Z}$ is the metal fraction) for masses above $0.1 \mathrm{M}_{\mathrm{Jup}}$ and the $\mathrm{Z}=0.5$ models for masses below $0.1 \mathrm{M}_{\mathrm{Jup}}$.

\subsection{Results}

Figure 3 shows the comparison of the instrumental sensitivities in terms of detectable companion mass for three different stellar types (G0, K0 and M0) as a function of their age. The left-hand side plot corresponds to the detection of planets at an angular separation of 0.2 " while the right-hand side one corresponds to an angular separation of 2". At 0.2", EPICS exploits at the bast the field of view of its $42 \mathrm{~m}$ telescope aperture while at the other end, MIRI is working at its very inner working angle. Logically, EPICS is therefore the most sensitive instrument for the smallest separation, no matter what the age or stellar type. Because of the relatively short integration time, the results obtained with EPICS are background limited. This is why the sensitivities are the same for the three stellar types. Between MIRI and SPHERE, MIRI is slightly better for the dimmest objects (M0 stars) but SPHERE is more efficient for G0 stars. Overall, we can show that the fainter and younger the star, the better the sensitivity in terms of companion mass. It explains why in this paper, we particularly focus on young M stars.

At 2" the situation is very different. First of all, we are well outside the EPICS FOV so that only SPHERE and MIRI can be used. Second, as we are at large angular separations from the star, none of the imagers are limited by the off-axis stellar residuals anymore but rather by the background level. Therefore, because MIRI is a space based telescope and because it operates at wavelengths where the star/planet contrast is more favorable, it is more sensitive with sensitivities down to $0.1 \mathrm{M}_{\mathrm{Jup}}$ for $10 \mathrm{Myr}$ old stars.

Figure 3 clearly illustrates the interest of observing young $\mathrm{M}$ stars rather than old stars with an earlier stellar type. Indeed, for MIRI at 0.2 ", the detection limit goes from $0.5 \mathrm{M}_{\mathrm{Jup}}$ around a $10 \mathrm{Myr}$ old M0 star to $3 \mathrm{M}_{\text {Jup }}$ planets around $1 \mathrm{Gyr}$ old G0 stars. Therefore, we decided to select a sample of M type stars among the closest and youngest moving groups. For all these targets, we compare the sensitivity of the three instruments. Figure 4 shows this comparison for the best targets in our sample. The number of observable stars with the two ground based facilities is lower because many targets are either too faint to be tracked by the AO systems $(V \leq 10)$ or not observable from these observatories. On this graph, two different sensitivities have been plotted for SPHERE: one with and one without SDI. The sensitivities with SDI clearly look better but we have to mitigate this first impression. Indeed, one must not forget that simulations with SDI implicitly assume that the planetary spectrum shows large variations within (at least one) of the spectral band(s) observed with SPHERE, e.g. $\mathrm{H} 2(1.593 \mu \mathrm{m})$ and $\mathrm{H} 3(1.667 \mu \mathrm{m})$ for the $\mathrm{H}$ band. If the magnitudes of cold planets changes significantly between these two spectral bands, it is not the case anymore for companions warmer than $\sim 500 \mathrm{~K}$. Therefore, some planets around $1 \mathrm{M}_{\text {Jup }}$ that should in principle be detectable with the SDI mode of SPHERE could be in fact below the detection limit due to their low SDI signature.

\section{PERSPECTIVES}

Direct imaging of planetary systems contributes to the understanding of planet formation while comparing photometry and evolutionary models for instance. By observing young $\mathrm{M}$ stars, it is possible to constrain theoretical models for the cooling of gas giants, especially at a few tens of Myr where the uncertainties and 

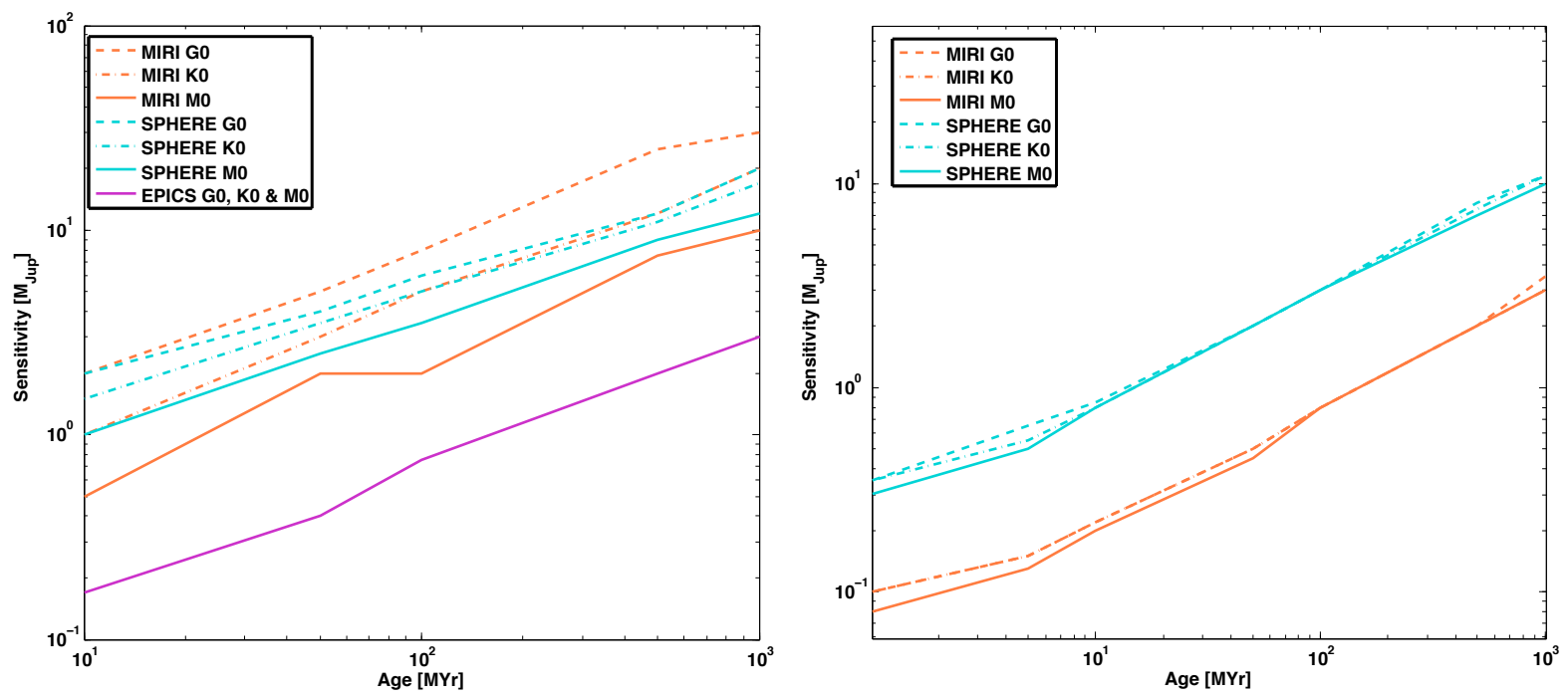

Figure 3. Left: Comparison of the instrumental sensitivities of SPHERE (with SDI), EPICS and MIRI at an angular separation of 0.2 " around G0, K0 and M0 stars as a function of the stellar age. Right: Same comparison at an angular separation of 2". Note that in this case, we are outside the EPICS field of view that cuts off at 0.4".
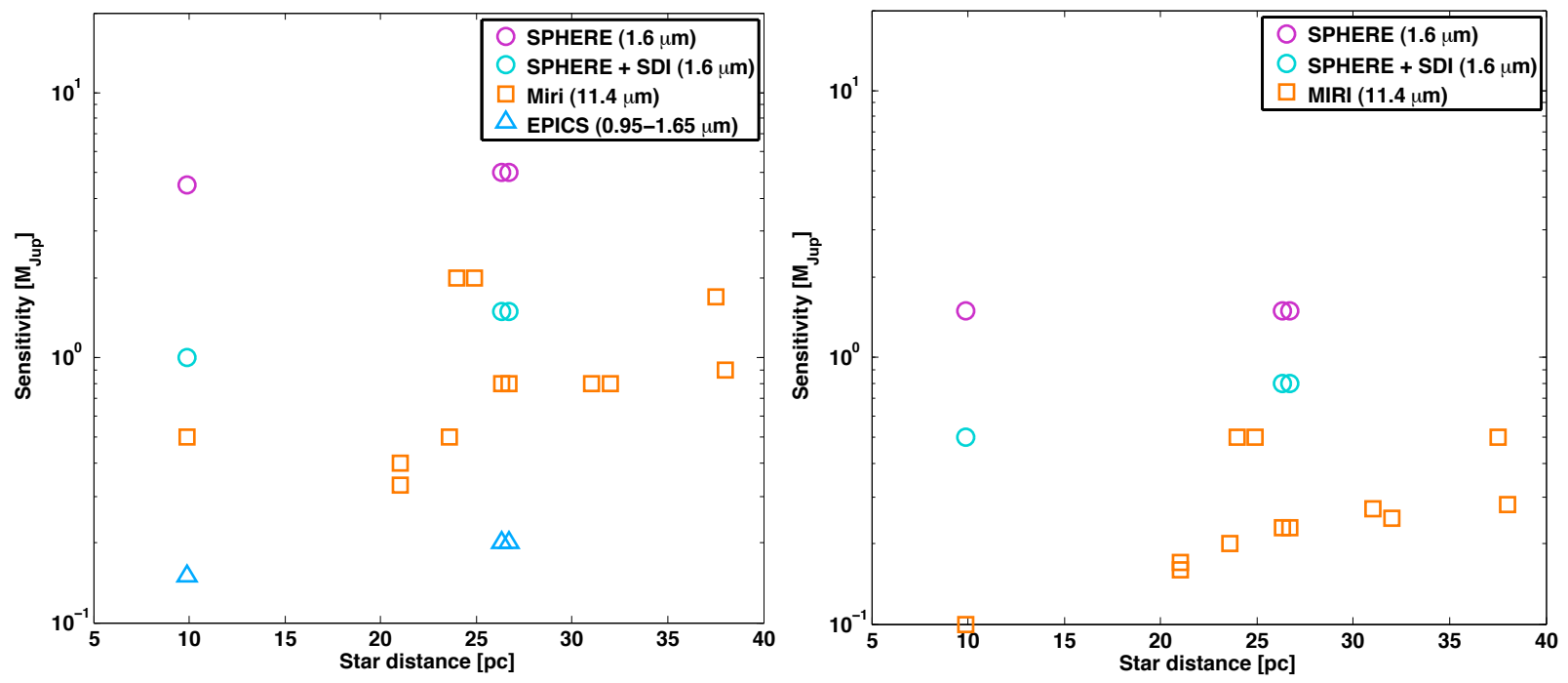

Figure 4. Left: Comparison of the instrumental sensitivities of SPHERE, EPICS and MIRI for an angular separation of 0.2 " around the closest M stars in young moving group associations as a function of the stellar distance. Right: Same comparison at an angular separation of 2 ". Note that in this case, we are outside the EPICS field of view that cuts off at $0.4 "$.

discrepancies between models are still large. To this aim, any discovery with the different planet imager facilities can be followed by means of other observations to perform photometric measurements at various wavelengths, using the MIRI FQPM modes at 10.65 and $15.5 \mu \mathrm{m}$ for example, as well as SPHERE observations in $\mathrm{H}$ or $\mathrm{K}$ bands. Follow-up observations of the most massive planets $\left(>1 \mathrm{M}_{\mathrm{Jup}}\right)$ found by such surveys will provide a direct distinction between the so-called "hot star" cooling models ${ }^{7}$ and the "core accretion" cooling models ${ }^{9}$ as they show discrepancies up to a few magnitudes during the first 10-100 Myr after their formation (see Figure 1). For more distant planets, it will also be possible to constrain the planetary orbit as well as the dynamical mass of the planets by taking images of the systems at two (or more) different orbital locations. Furthermore, it will provide first constraints on the models in a very interesting mass regime where the planetary radius is expected 


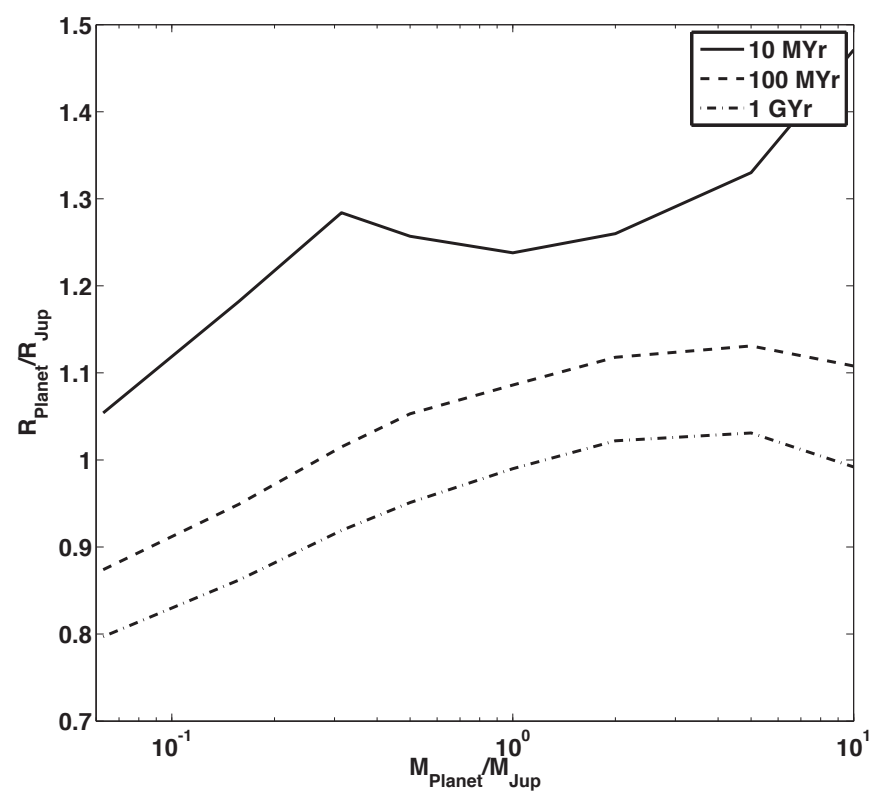

Figure 5. Mass-radius relationship from the Baraffe et al. (2008) ${ }^{30}$ model at metallicity $\mathrm{Z}=0.10$ for three different ages. JWST/MIRI has the potential to probe for the first time the peak appearing at $\mathrm{M} \sim 0.3$ Mjup in the 10 Myr model. Note that such discontinuities also appear for other metallicities, and are also predicted by the models of Fortney et al. $(2008)^{9}$ for a similar mass range.

to increase for decreasing masses (see Figure 5). Photometric measurements with MIRI alone will provide a model-independent estimation of the planetary temperature by comparing the flux at 11.4 and $15.5 \mu \mathrm{m}$ with a blackbody emission, which can directly be used to constrain the cooling models. MIRI observations at $10.65 \mu \mathrm{m}$ will allow us to detect the presence of ammonia through its absorption band at that particular wavelength, which could also be used to further constrain the planetary temperature. Follow-up observations with SPHERE in $\mathrm{H}$ and K bands (for planets closer than 5") or EPICS between 0.95 and $1.65 \mu \mathrm{m}$ (for planets within 0.4") would also greatly help in constraining theoretical emergent spectra predicted by various atmospheric models. Finally, for planets at short enough periods $(<20 \mathrm{yrs})$, follow-up astrometric and/or RV observations could provide a model-independent estimation of the planetary mass.

\section{CONCLUSION}

From the comparison of performances for exoplanet detection that we have presented in this paper, we have identified a catalogue of targets which are particularly well suited for each instrument. Our study identifies a very interesting niche for the MIRI instrument onboard JWST. This niche consists in the observation of nearby M-stars located in young moving groups. Indeed in most cases, such M-stars are too faint to be used as guide stars for the (extreme) adaptive optics systems that will equip future ground-based planet imagers. Therefore, only MIRI will provide a good sensitivity to faint companions around these targets, down to Neptune-mass planets in the most favorable cases. We also show that for earlier stellar types (i.e. A, F, G and K), SPHERE and EPICS will be on par with, or will outperform MIRI. However, even for the earlier stellar types, MIRI will be the only instrument capable of taking spectra of Jupiter mass planets in the mid-IR. We therefore conclude that these three instruments are very complimentary for exoplanet imaging as they will probe different angular separations and will be optimal for different types of stars.

\section{ACKNOWLEDGMENTS}

This research was supported by the Fond National de la Recherche scientifique de Belgique (FNRS), by the Fonds pour la formation à la Recherche dans l'Industrie et dans l'Agriculture de Belgique (FRIA) and by the 
Communauté Francaise de Belgique - Action de recherche concertée - Académie Wallonie - Europe. The authors acknowledge Christophe Verinaud for providing us the coronagraphic profiles of EPICS.

\section{REFERENCES}

[1] Marois, C., Macintosh, B., Barman, T., Zuckerman, B., Song, I., Patience, J., Lafrenière, D., and Doyon, R., "Direct Imaging of Multiple Planets Orbiting the Star HR 8799," Science 322, 1348- (Nov. 2008).

[2] Kalas, P., Graham, J. R., Chiang, E., Fitzgerald, M. P., Clampin, M., Kite, E. S., Stapelfeldt, K., Marois, C., and Krist, J., "Optical Images of an Exosolar Planet 25 Light-Years from Earth," Science 322, 1345(Nov. 2008).

[3] Serabyn, E., Mawet, D., and Burruss, R., "An image of an exoplanet separated by two diffraction beamwidths from a star," Nature 464, 1018-1020 (Apr. 2010).

[4] Lagrange, A., Gratadour, D., Chauvin, G., Fusco, T., Ehrenreich, D., Mouillet, D., Rousset, G., Rouan, D., Allard, F., Gendron, É., Charton, J., Mugnier, L., Rabou, P., Montri, J., and Lacombe, F., "A probable giant planet imaged in the $\beta$ Pictoris disk. VLT/NaCo deep L'-band imaging," A $8 A$ 493, L21-L25 (Jan. 2009).

[5] Udry, S. and Santos, N. C., "Statistical Properties of Exoplanets," ARA\&A 45, 397-439 (Sept. 2007).

[6] Lagrange, A., Desort, M., Galland, F., Udry, S., and Mayor, M., "Extrasolar planets and brown dwarfs around A-F type stars. VI. High precision RV survey of early type dwarfs with HARPS," A\&SA 495, 335352 (Feb. 2009).

[7] Baraffe, I., Chabrier, G., Barman, T. S., Allard, F., and Hauschildt, P. H., "Evolutionary models for cool brown dwarfs and extrasolar giant planets. The case of HD 209458," A\&A 402, 701-712 (May 2003).

[8] Marley, M. S., Fortney, J. J., Hubickyj, O., Bodenheimer, P., and Lissauer, J. J., "On the Luminosity of Young Jupiters," ApJ 655, 541-549 (Jan. 2007).

[9] Fortney, J. J., Marley, M. S., Saumon, D., and Lodders, K., "Synthetic Spectra and Colors of Young Giant Planet Atmospheres: Effects of Initial Conditions and Atmospheric Metallicity," ApJ 683, 1104-1116 (Aug. 2008).

[10] Zuckerman, B. and Song, I., "Young Stars Near the Sun," ARA\&A 42, 685-721 (Sept. 2004).

[11] Beuzit, J., Feldt, M., Dohlen, K., Mouillet, D., Puget, P., Wildi, F., Abe, L., Antichi, J., Baruffolo, A., Baudoz, P., Boccaletti, A., Carbillet, M., Charton, J., Claudi, R., Downing, M., Fabron, C., Feautrier, P., Fedrigo, E., Fusco, T., Gach, J., Gratton, R., Henning, T., Hubin, N., Joos, F., Kasper, M., Langlois, M., Lenzen, R., Moutou, C., Pavlov, A., Petit, C., Pragt, J., Rabou, P., Rigal, F., Roelfsema, R., Rousset, G., Saisse, M., Schmid, H., Stadler, E., Thalmann, C., Turatto, M., Udry, S., Vakili, F., and Waters, R., "SPHERE: a planet finder instrument for the VLT," in [Society of Photo-Optical Instrumentation Engineers (SPIE) Conference Series], Presented at the Society of Photo-Optical Instrumentation Engineers (SPIE) Conference $\mathbf{7 0 1 4}$ (Aug. 2008).

[12] Macintosh, B. A., Graham, J. R., Palmer, D. W., Doyon, R., Dunn, J., Gavel, D. T., Larkin, J., Oppenheimer, B., Saddlemyer, L., Sivaramakrishnan, A., Wallace, J. K., Bauman, B., Erickson, D. A., Marois, C., Poyneer, L. A., and Soummer, R., "The Gemini Planet Imager: from science to design to construction," in [Society of Photo-Optical Instrumentation Engineers (SPIE) Conference Series], Presented at the Society of Photo-Optical Instrumentation Engineers (SPIE) Conference 7015 (July 2008).

[13] Roberts, L. C., Shao, M., Vasisht, G., Levine, B. M., Aguayo, F. F., Nichols, J., Lockhart, T. G., Knight, H., Oppenheimer, B. R., and Hinkley, S., "Exoplanet Imaging at the Palomar 5-m: Enhancing the Contrast of the Project 1640 Coronagraph," in [American Astronomical Society Meeting Abstracts], American Astronomical Society Meeting Abstracts 214 (May 2009).

[14] Nelson, J. and Sanders, G. H., "TMT status report," in [Society of Photo-Optical Instrumentation Engineers (SPIE) Conference Series], Presented at the Society of Photo-Optical Instrumentation Engineers (SPIE) Conference $\mathbf{6 2 6 7}$ (July 2006).

[15] Johns, M., "Progress on the GMT," in [Society of Photo-Optical Instrumentation Engineers (SPIE) Conference Series], Presented at the Society of Photo-Optical Instrumentation Engineers (SPIE) Conference 7012 (Aug. 2008). 
[16] Markus, K., "Overall science goals and top level AO requirements for the E-ELT," in [Adaptative Optics for Extremely Large Telescopes], (2010).

[17] Wright, G. S., Rieke, G. H., Colina, L., van Dishoeck, E., Goodson, G., Greene, T., Lagage, P., Karnik, A., Lambros, S. D., Lemke, D., Meixner, M., Norgaard, H., Oloffson, G., Ray, T., Ressler, M., Waelkens, C., Wright, D., and Zhender, A., "The JWST MIRI instrument concept," in [Society of Photo-Optical Instrumentation Engineers (SPIE) Conference Series], J. C. Mather, ed., Presented at the Society of PhotoOptical Instrumentation Engineers (SPIE) Conference 5487, 653-663 (Oct. 2004).

[18] Rieke, M. J., Baum, S. A., Beichman, C. A., Crampton, D., Doyon, R., Eisenstein, D., Greene, T. P., Hodapp, K., Horner, S. D., Johnstone, D., Lesyna, L., Lilly, S., Meyer, M., Martin, P., McCarthy, Jr., D. W., Rieke, G. H., Roellig, T. L., Stauffer, J., Trauger, J. T., and Young, E. T., "NGST NIRCam Scientific Program and Design Concept," in [Society of Photo-Optical Instrumentation Engineers (SPIE) Conference Series], J. C. Mather, ed., Presented at the Society of Photo-Optical Instrumentation Engineers (SPIE) Conference 4850, 478-485 (Mar. 2003).

[19] Doyon, R., Rowlands, N., Hutchings, J., Evans, C. E., Greenberg, E., Scott, A. D., Touhari, D., Beaulieu, M., Abraham, R., Ferrarese, L., Fullerton, A. W., Jayawardhana, R., Johnston, D., Meyer, M. R., Pipher, J., and Sawicki, M., "The JWST tunable filter imager (TFI)," in [Society of Photo-Optical Instrumentation Engineers (SPIE) Conference Series], Presented at the Society of Photo-Optical Instrumentation Engineers (SPIE) Conference $\mathbf{7 0 1 0}$ (Aug. 2008).

[20] Riaud, P., Boccaletti, A., Rouan, D., Lemarquis, F., and Labeyrie, A., "The Four-Quadrant Phase-Mask Coronagraph. II. Simulations," PASP 113, 1145-1154 (Sept. 2001).

[21] Riaud, P., Boccaletti, A., Baudrand, J., and Rouan, D., "The Four-Quadrant Phase Mask Coronagraph. III. Laboratory Performance," PASP 115, 712-719 (June 2003).

[22] Soummer, R., "Apodized Pupil Lyot Coronagraphs for Arbitrary Telescope Apertures," ApJ 618, L161L164 (Jan. 2005).

[23] Boccaletti, A., Abe, L., Baudrand, J., Daban, J., Douet, R., Guerri, G., Robbe-Dubois, S., Bendjoya, P., Dohlen, K., and Mawet, D., "Prototyping coronagraphs for exoplanet characterization with SPHERE," in [Society of Photo-Optical Instrumentation Engineers (SPIE) Conference Series], Society of Photo-Optical Instrumentation Engineers (SPIE) Conference Series 7015 (July 2008).

[24] Kasper, M. E., Beuzit, J., Verinaud, C., Yaitskova, N., Baudoz, P., Boccaletti, A., Gratton, R. G., Hubin, N., Kerber, F., Roelfsema, R., Schmid, H. M., Thatte, N. A., Dohlen, K., Feldt, M., Venema, L., and Wolf, S., "EPICS: the exoplanet imager for the E-ELT," in [Society of Photo-Optical Instrumentation Engineers (SPIE) Conference Series], Society of Photo-Optical Instrumentation Engineers (SPIE) Conference Series 7015 (July 2008).

[25] Vérinaud, C., Korkiakoski, V., Yaitskova, N., Martinez, P., Kasper, M. E., Beuzit, J., Abe, L., Baudoz, P., Boccaletti, A., Dohlen, K., Gratton, R. G., Mesa, D., Kerber, F., Schmid, H. M., Venema, L., Salter, G., Tecza, M., and Thatte, N. A., "System design and analysis of the exo-planet imaging camera and spectrograph (EPICS) for the European ELT," in [Society of Photo-Optical Instrumentation Engineers (SPIE) Conference Series], Society of Photo-Optical Instrumentation Engineers (SPIE) Conference Series 7014 (Aug. 2008).

[26] Boccaletti, A., Baudoz, P., Baudrand, J., Reess, J. M., and Rouan, D., "Imaging exoplanets with the coronagraph of JWST/MIRI," Advances in Space Research 36, 1099-1106 (2005).

[27] Baudoz, P., Boccaletti, A., Riaud, P., Cavarroc, C., Baudrand, J., Reess, J. M., and Rouan, D., "Feasibility of the Four-Quadrant Phase Mask in the Mid-Infrared on the James Webb Space Telescope," PASP 118, 765-773 (May 2006).

[28] Lenzen, R., Close, L., Brandner, W., Hartung, M., and Biller, B., "NACO-SDI: A Novel Simultaneous Differential Imager for the Direct Imaging of Giant Extra-Solar Planets," in [Science with Adaptive Optics], Brandner, W. and Kasper, M. E., eds., 46 (2005).

[29] Carbillet, M., Boccaletti, A., Thalmann, C., Fusco, T., Vigan, A., Smith, I., Mouillet, D., Dohlen, K., Bendjoya, P., and Ferrari, A., "The Software Package SPHERE: a CAOS-based numerical tool for end-to-end simulations of SPHERE/VLT," in [Society of Photo-Optical Instrumentation Engineers (SPIE) Conference Series], Society of Photo-Optical Instrumentation Engineers (SPIE) Conference Series 7015 (July 2008). 
[30] Baraffe, I., Chabrier, G., and Barman, T., "Structure and evolution of super-Earth to super-Jupiter exoplanets. I. Heavy element enrichment in the interior," $A \mathscr{E} A$ 482, 315-332 (Apr. 2008). 